\title{
Learning Unknown from Correlations: Graph Neural Network for Inter-novel-protein Interaction Prediction
}

\author{
Guofeng Lv，Zhiqiang Hu，Yanguang Bi，Shaoting Zhang \\ SenseTime Research \\ \{lvguofeng, huzhiqiang, biyanguang, zhangshaoting $\} @$ sensetime.com
}

\begin{abstract}
The study of multi-type Protein-Protein Interaction (PPI) is fundamental for understanding biological processes from a systematic perspective and revealing disease mechanisms. Existing methods suffer from significant performance degradation when tested in unseen dataset. In this paper, we investigate the problem and find that it is mainly attributed to the poor performance for inter-novel-protein interaction prediction. However, current evaluations overlook the inter-novel-protein interactions, and thus fail to give an instructive assessment. As a result, we propose to address the problem from both the evaluation and the methodology. Firstly, we design a new evaluation framework that fully respects the inter-novel-protein interactions and gives consistent assessment across datasets. Secondly, we argue that correlations between proteins must provide useful information for analysis of novel proteins, and based on this, we propose a graph neural network based method (GNN-PPI) for better inter-novel-protein interaction prediction. Experimental results on real-world datasets of different scales demonstrate that GNN-PPI significantly outperforms state-of-the-art PPI prediction methods, especially for the inter-novel-protein interaction prediction. ${ }^{1}$
\end{abstract}

\section{Introduction}

Protein-protein Interactions (PPIs) play an important role in most biological processes. In addition to direct physical binding, PPI also has many other, indirect ways of cooperation and mutual regulation, such as exchange reaction products, participate in signal relay mechanisms, or jointly contribute toward specific organismal functions [Szklarczyk et al., 2016]. It can be said that the study of PPIs and their interaction types are essential toward understanding cellular biological processes in normal and disease states, which in turn facilitate the therapeutic target identification and novel drug

\footnotetext{
${ }^{1}$ Codes are available at https://github.com/lvguofeng/GNN_PPI Extended version https://arxiv.org/abs/2105.06709
}

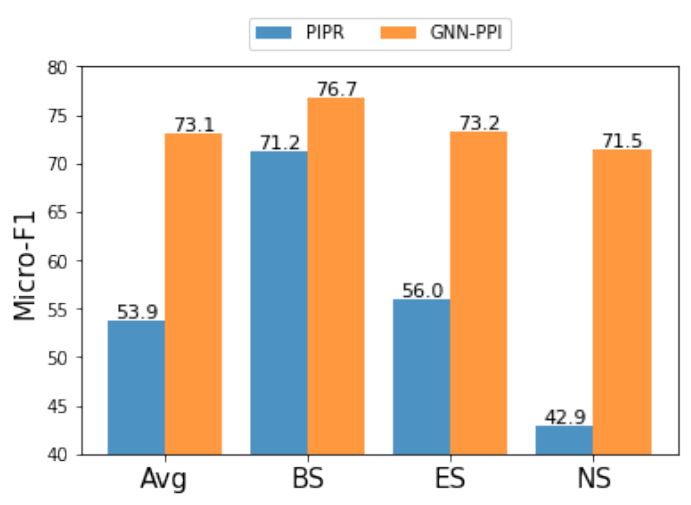

Figure 1: Results of PIPR (baseline) and GNN-PPI (ours) when trained on the smaller dataset SHS148k and tested on the larger STRING dataset. The metric is micro F1 score for multi-label PPI type prediction. Avg is the overall result of the testset. For further investigation, we divide the testset into BS, ES and NS subsets, where BS denotes Both of the pair proteins in interaction were Seen during training, ES denotes Either (but not both) of the pair proteins was Seen, and NS denotes Neither proteins were Seen during training. We regard ES and NS as inter-novel-protein interactions.

design [Skrabanek et al., 2008]. There are many experimental methods to detect PPI, where the most conventional and widely used high-throughput methods are yeast two-hybrid screening [Fields and Song, 1989]. However, the experimentbased methods are expensive and time-consuming, but more importantly, even if a single experiment has detected PPI, it cannot fully interpret its types [De Las Rivas and Fontanillo, 2010]. Evidently, we urgently need reliable computational methods that are learned from the accumulated PPI data to predict the unknown PPIs accurately.

Despite long-term research works [Guo et al., 2008; Hashemifar et al., 2018; Chen et al., 2019] make noticeable progress, existing methods suffer from significant performance degradation when tested on unseen dataset. Take the state-of-the-art model PIPR [Chen et al., 2019] as an example, compared tested on trainset-homologous SHS148k testset with on a larger STRING testset, micro F1 score drops from 92.42 to 53.85. For further investigation, we divide the STRING testset into BS, ES and NS subsets, where BS denotes Both of the pair proteins in interaction were Seen during 
training, ES denotes Either (but not both) of the pair proteins was Seen, and NS denotes Neither proteins were Seen during training. As clearly shown in Figure 1, poor performance in the ES and NS subsets (collectively termed as inter-novelprotein interactions in this paper) is the main reason for the performance degradation.

On the other hand, current evaluations on the trainsethomologous SHS148k testset apply a protein-irrepective perinteraction randomized strategy to divide the trainset and testset, and consequently, BS comprises over $92 \%$ of the whole testset $^{1}$ and dominates the overall performance. The evaluations overlook the inter-novel-protein interactions, and are thus not instructive for the performance when tested on other datasets. As a result, in this paper we firstly design a new evaluation framework with two per-protein randomized data partition strategies. Instead of simple protein-independent randomization, we take also into consideration the distance between proteins and utilize Breadth-First and Depth-First Search methods to construct the testset. Comparison experiments between the trainset-homologous testset and the unseen STRING testset demonstrates the proposed evaluation can give consistent assessment across datasets.

Besides the evaluation, for the methodology existing works take PPIs as independent instances. Correlations between proteins have long been ignored. Intuitively, for predicting the type of interaction between protein $\mathrm{A}$ and $\mathrm{B}$, the interaction between protein $\mathrm{A}$ and $\mathrm{C}$, as well as $\mathrm{B}$ and $\mathrm{C}$ must provide useful information. The correlations can be naturally modeled and excavated with a graph, where proteins serve as the nodes and interactions as the edges. In this paper, the graph is processed with a graph neural network based model (GNN-PPI). As demonstrated in Figure 1, the introduction of correlations and the proposed GNN-PPI model have largely narrow the performance gap between BS, ES and NS subsets.

In summary, the contribution of this paper is three-fold:

1. We design a new evaluation framework that fully respects the inter-novel-protein interactions and give consistent assessment across datasets.

2. We propose to incorporate correlation between proteins into the PPI prediction problem. A graph neural network based method is presented to model the correlations.

3. The proposed GNN-PPI model achieves state-of-the-art performance in real datasets of different scales, especially for the inter-novel-protein interaction prediction.

\section{Related Work}

The primary amino acid sequences are confirmed to contain all the protein information [Anfinsen, 1972] and are extremely easy to obtain. Thus, there is a longstanding interest in using sequence-based methods to model proteinrelated tasks. The research work of PPI prediction and classification can be summarized into two stages. The early research is based on Machine Learning (ML) [Guo et al., 2008; Wong et al., 2015; Silberberg et al., 2014; Shen et al., 2007]. These methods provide feasible solutions, but their performance is limited by the PPI feature representation and model expressiveness. Deep Learning (DL) has recently been widely used in bioinformatics problems due to its powerful expressive ability, including PPI prediction and classification. These works [Li et al., 2018; Hashemifar et al., 2018; Chen et al., 2019; Sun et al., 2017] typically use Convolution Neural Networks or Recurrent Neural Networks to extract features from the amino acid sequence of the protein.

More recent work has focused on the feature representation of proteins. [Saha and others, 2020] proposes a novel deep multi-modal architecture, which extracts multi-modal information from protein structure and existing text information in biomedical literature. [Nambiar et al., 2020] proposes a Transformer based neural network to generate proteins pretrained embedding. In the latest research, [Yang et al., 2020] considers the correlation of PPIs and first proposed to use GCN[Kipf and Welling, 2016] to learn protein features in the PPI network automatically. However, their work cannot be extended to the multi-label PPI classification.

To the best of our knowledge, the current work of PPI has not been concerned with the problems of inter-novel-protein interactions. However, In the field of Drug-drug Interaction (DDI), [Deng et al., 2020] mentioned that the testset is divided according to whether the drug was seen during training, and the results show that the performance for the inter-noveldrug interactions is extremely degraded, but the original paper does not propose a solution.

\section{Methodology}

\subsection{Problem Formulation}

Suppose we have protein set $\mathcal{P}=\left\{p_{0}, p_{1}, \ldots, p_{n}\right\}$ and PPI set $\mathcal{X}=\left\{x_{i j}=\left\{p_{i}, p_{j}\right\} \mid i \neq j, p_{i}, p_{j} \in \mathcal{P}, I\left(x_{i j}\right) \in\{0,1\}\right\} . I$ is the PPI indicator function, if $I\left(x_{i j}\right)=1$, then it means that protein $p_{i}$ interacts with protein $p_{j}$. Note that when $I\left(x_{i j}\right)=0$, it may mean that protein $p_{i}$ and $p_{j}$ will not interact, or they have a potential interaction while it has not been discovered so far. In order to avoid unnecessary errors, we will not do any operation on unknown protein pairs (default $\forall x_{i j} \in \mathcal{X}, I\left(x_{i j}\right)=1$ ). We define PPI label space as $\mathcal{L}=\left\{l_{0}, l_{1}, \ldots, l_{n}\right\}$ with $n$ possible interaction types. For each PPI $x_{i j}$, its labels is represented as $y_{i j} \subseteq \mathcal{L}$. In summary, the multi-type PPI dataset is defined as $\mathcal{D}=\left\{\left(x_{i j}, y_{i j}\right) \mid x_{i j} \in \mathcal{X}\right\}$. Considering the correlation of PPIs, we use protein as nodes and PPIs as edges to build the PPI graph $\mathcal{G}=(\mathcal{P}, \mathcal{X})$.

The task of multi-type PPI learning is to learn a model $\mathcal{F}: x \rightarrow \hat{y}$ from the training set $\mathcal{X}_{\text {train. }}$. For any protein pair $x_{i j} \in \mathcal{X}_{\text {test }}$, the model $\mathcal{F}$ predict $\hat{y}_{i j}$ as the set of proper labels for $x_{i j}$. The above-mentioned $\mathcal{X}_{\text {train }}$ and $\mathcal{X}_{\text {test }}$ are obtained from $\mathcal{X}$ based on the evaluation, where $\mathcal{X}_{\text {train }}+\mathcal{X}_{\text {test }}=\mathcal{X}$. Further, according to whether protein $p$ was seen during training, the protein set $\mathcal{P}$ is divided into known $\mathcal{P}_{v}=\bigcup \mathcal{X}_{\text {train }}$ and unknown $\mathcal{P}_{u}=\mathcal{P}-\mathcal{P}_{\text {train }}$. Moreover, as mentioned in section $1, \mathcal{X}_{\text {test }}$ can be divided into $\mathcal{X}_{\mathrm{BS}}, \mathcal{X}_{\mathrm{ES}}$, and $\mathcal{X}_{\mathrm{NS}}$, which defined as follows:

$$
\begin{aligned}
& \mathcal{X}_{\mathrm{BS}}=\left\{x_{i j} \mid x_{i j} \in \mathcal{X}_{\text {test }}, p_{i}, p_{j} \in \mathcal{P}_{v}\right\} \mathcal{X}_{\mathrm{ES}}=\left\{x_{i j} \mid x_{i j} \in \mathcal{X}_{\text {test }}, p_{i} \in \mathcal{P}_{u}, p_{j} \in \mathcal{P}_{v}\right. \\
&\left.\quad \text { or } p_{j} \in \mathcal{P}_{u}, p_{i} \in \mathcal{P}_{v}\right\} \\
& \mathcal{X}_{\mathrm{NS}}=\left\{x_{i j} \mid x_{i j} \in \mathcal{X}_{\text {test }}, p_{i}, p_{j} \in \mathcal{P}_{u}\right\}
\end{aligned}
$$




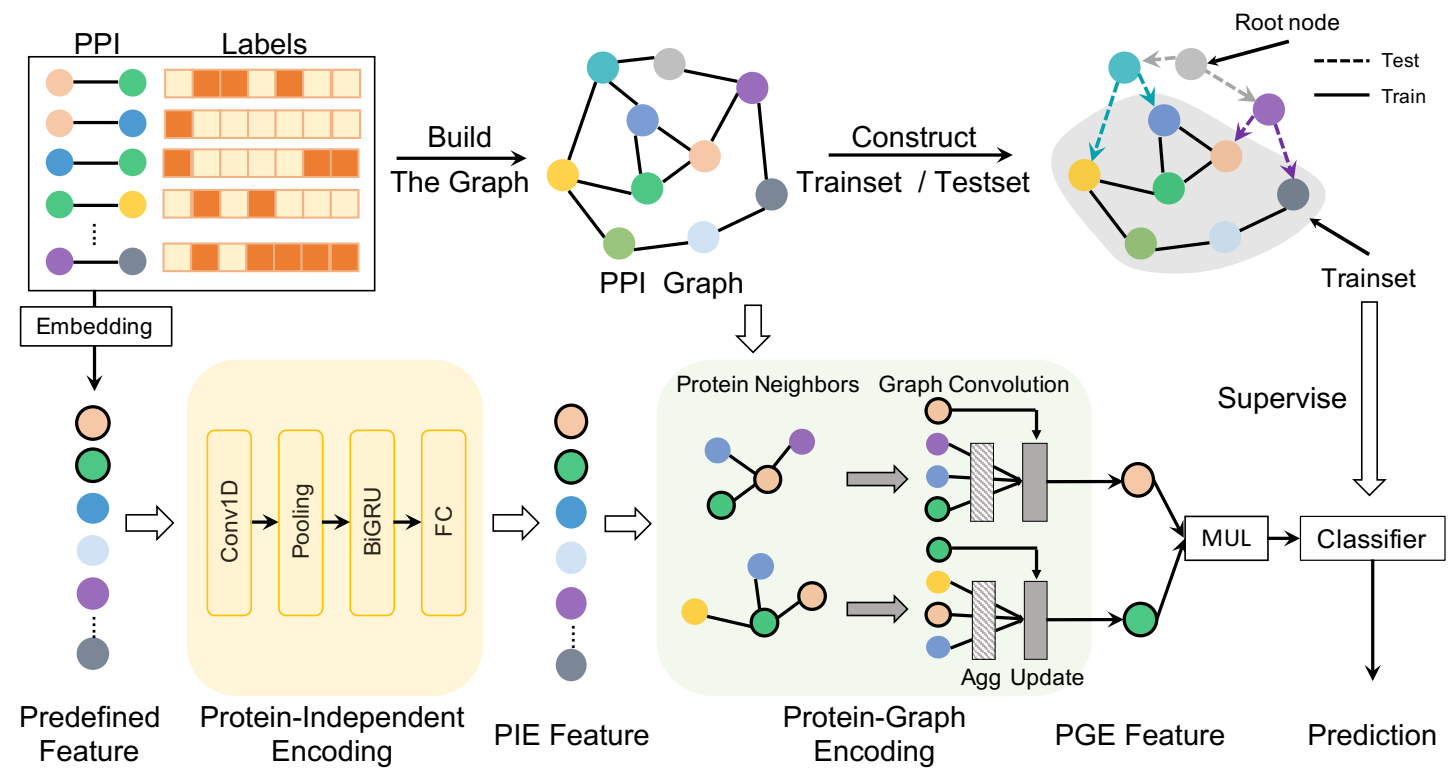

Figure 2: Development and evaluation of the GNN-PPI framework. Pairwise interaction data are firstly assembled to build the graph, where proteins serve as the nodes and interactions as the edges. The testset is constructed by firstly selecting the root node and then performing the proposed BFS or DFS strategy. The model is developed by firstly performing embedding for each protein to obtain predefined features, then processed by Convolution, Pooling, BiGRU and FC modules to extract protein-independent encoding (PIE) features, which are finally aggregated by graph convolutions and arrive at protein-graph encoding (PGE) features. Features of the pair proteins in interaction are multiplied and classified, supervised by the trainset labels.

Since inter-novel-protein interactions are the main bottlenecks, we require the testset $\mathcal{X}_{\text {test }}$ of the evaluation framework to meet condition $\left|\mathcal{X}_{\mathrm{BS}}\right| \ll\left|\mathcal{X}_{\mathrm{ES}}\right|+\left|\mathcal{X}_{\mathrm{NS}}\right|$. Our goal is that under this evaluation, the model $\mathcal{F}$ learned from $\mathcal{X}_{\text {train }}$ can accurately predict the multi-label label of PPI in $\mathcal{X}_{\text {test }}$.

\subsection{Overview}

The GNN-PPI framework and evaluation are shown in Figure 2. We will introduce GNN-PPI from the following three aspects. First is Evaluation Framework. We propose two sets of heuristic data partition schemes based on the PPI network, and the generated testset meets the conditions $\left|\mathcal{X}_{\mathrm{BS}}\right| \ll$ $\left|\mathcal{X}_{\mathrm{ES}}\right|+\left|\mathcal{X}_{\mathrm{NS}}\right|$. Secondly, Protein feature encoding. We design Protein-Independent Encoding (PIE) and Protein-Graph Encoding (PGE) modules to encode protein features. The last is Multi-label PPI prediction. For unknown PPIs, we combine their protein feature encoded by the previous process, calculate their scores in different PPI types, and output its multi-label prediction.

\subsection{Evaluation Framework}

Generally, existing machine learning algorithms usually randomly divide part of the dataset as a testset to evaluate the performance of the model. However, in the PPI-related tasks, we have the following Corollary, derived from Erdős-Rényi(ER) random graph model. [ERDdS and R\&wi, 1959; Erdôs and Rényi, 1960]:

Corollary 1. Randomly divide the PPI dataset, select $t \leq 0.2$ as the testset, then most of the proteins in the testset were seen in training.

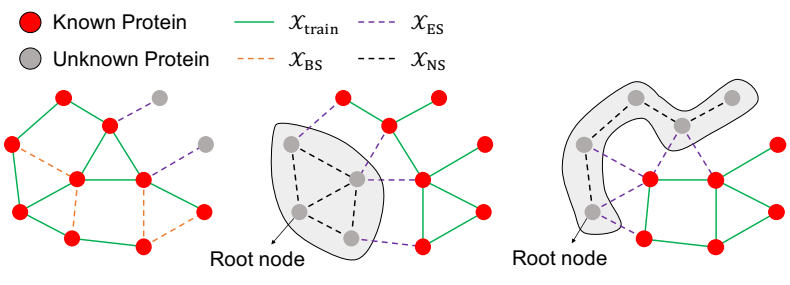
(a) Random
(b) BFS
(c) DFS

Figure 3: Examples of different testset construction strategies. Random is the current scheme, while Breath-First Search (BFS) and Depth-First Search (DFS) are the proposed schemes.

The detailed proof of the corollary is elaborated in the extended version ${ }^{1}$. It can be inferred from this corollary that the performance of the testset obtained by random division only reflects the predictive ability of the PPI between known $\mathcal{P}_{v}$. In the real world, there are still many proteins and their PPIs that have not been discovered. We perform empirical studies by comparison of two different time points, 2021/01/25 and 2020/04/11, of the Homo sapiens subset of BioGRID database. We found that the newly discovered proteins exhibit some BFS-like or DFS-like local patterns. Even if the PPIs have been discovered, most of their types remain relatively unexplored. Therefore, we need a brand-new evaluation that can reflect the model's predictive performance on the internovel-protein interactions. The next content will introduce the evaluation framework we design.

We design two heuristic evaluation schemes based on the 


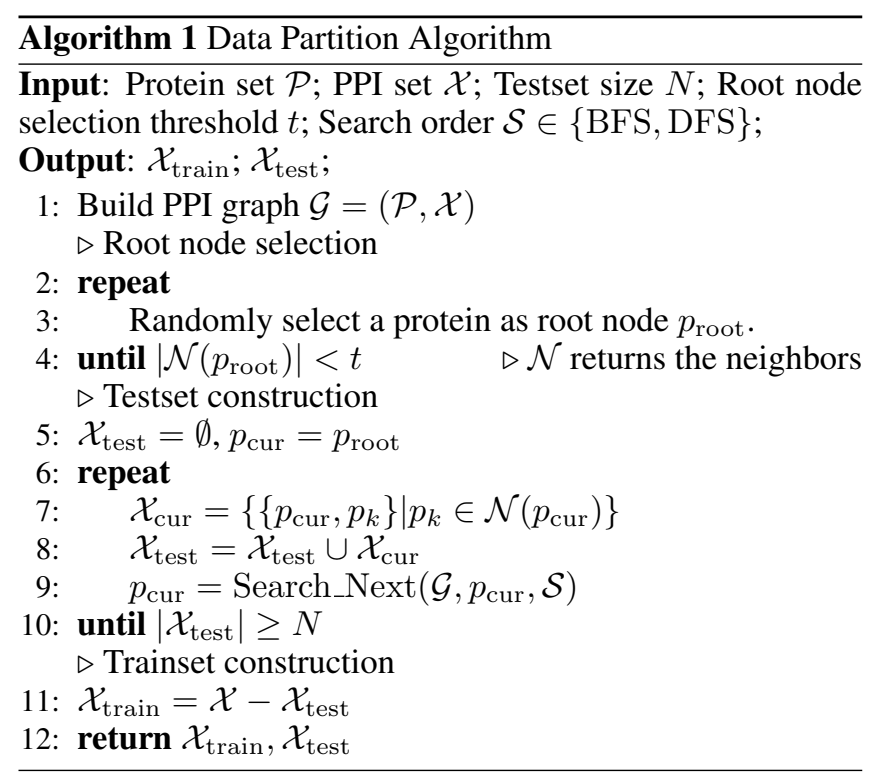

PPI network, namely BFS and DFS. They simulated two scenarios of unknown proteins $\mathcal{P}_{u}$ in reality:

1. $\mathcal{P}_{u}$ interact tightly with each other, and they exist in the form of clusters in the PPI network (See Figure 3 (b)).

2. $\mathcal{P}_{u}$ are sparsely distributed in the PPI network and have little interaction with each other (See Figure 3 (c)).

We select a root node $p_{\text {root }}$, fix the size of the testset $N$, and then use the Breadth-First Search (BFS) algorithm in the PPI network to obtain the proteins $\mathcal{P}_{u}$ that meet the scenario 1 . All PPIs related to these proteins are the generated testset. For scenario 2, we just need to simply randomly select proteins to form $\mathcal{P}_{u}$. However, in order to maintain the PPI network connectivity of $\mathcal{X}_{\text {train }}$ and $\mathcal{X}_{\text {test }}$, we use the Depth-First Search (DFS) algorithm to simulate. The details of the data partition algorithm are shown in Algorithm 1, where we will not show the details of the BFS and DFS algorithms but use the Search_Next function to return the next protein of the current protein $p_{\text {cur }}$ in different search algorithms. The $\mathcal{N}(p)$ returns all neighbors of protein $p$. And we controls the degree of the root node $\left|\mathcal{N}\left(p_{\text {root }}\right)\right|<t$ to simulate newly discovered proteins (Usually few proteins interact with them).

\subsection{Protein Feature Encoding}

Previous work [Chen et al., 2019] has proved that protein features based on the amino acid sequence are beneficial to the performance improvement of PPI-related tasks. Therefore, we design a Protein-Independent Encoding (PIE) module, which contains Conv1d with pooling, BiGRU, and fully connected (FC) layer, to generate protein feature representations as input to the PPI network.

The subsequent Protein-Graph Encoding (PGE) module is the core of GNN-PPI. Inspired by PPI network being widely used in bioinformatics computing, we construct PPI network $\mathcal{G}=(\mathcal{P}, \mathcal{X})$, and convert the original independent learning tasks $\mathcal{F}\left(x_{i j} \mid p_{i}, p_{j}, \theta\right) \rightarrow \hat{y}_{i j}$ into graph-related learning tasks $\mathcal{F}\left(x_{i j} \mid \mathcal{G}, \theta\right) \rightarrow \hat{y}_{i j}$. Recently, GNN is the most effective graph representation learning method, its main idea is the recursive neighborhood aggregation scheme, where each node computes a new feature by aggregating the previous features of its neighbor nodes. After $k$ iterations, a node is represented by its transformed feature, which captures the structural information within the node's $k$-hop neighborhood. More specifically, the GNN of the k-th iteration is

$a_{p}^{k}=\operatorname{Agg}\left(\left\{g_{p^{\prime}}^{k-1} \mid p^{\prime} \in \mathcal{N}(p)\right\}\right), g_{p}^{k}=\operatorname{Update}\left(\left\{g_{p}^{k-1}, a_{p}^{k}\right\}\right)$ where $g_{p}^{k}$ is the feature of node $p$ at the k-th iteration. The design of $\operatorname{Agg}(\cdot)$ and Update $(\cdot)$ are the keys to different GNN architectures. In this paper we use Graph Isomorphism Network (GIN) [Xu et al., 2018], where the sum of the neighbor node features is used as the aggregation function, and multilayer perceptrons (MLPs) is used to update the aggregated features. Then, GIN updates node features as

$$
g_{p}^{k}=\operatorname{MLP}^{k}\left(\left(1+\epsilon^{k}\right) \cdot g_{p}^{k-1}+\sum_{p^{\prime} \in \mathcal{N}(p)} g_{p^{\prime}}^{k-1}\right)
$$

where $\epsilon$ can be a learnable parameter or a fixed scalar.

\subsection{Multi-Label PPI Prediction}

With the feature of protein learned from the previous stages for the PPI $x_{i j}$, we use the dot product operation to combine the features of $p_{i}$ and $p_{j}$, and then use a fully connected layer (FC) as classifier for multi-label PPI prediction, expressed as $\hat{y}_{i j}=\mathrm{FC}\left(g_{p_{i}} \cdot g_{p_{j}}\right)$. The PIE and PGE modules are jointly training in an end-to-end way. Given a training set $\mathcal{X}_{\text {train }}$ and its ground-truth multi-label interaction $\mathcal{Y}_{\text {train }}$, we can use the multi-task binary cross-entropy as the loss function:

$L=\sum_{k=0}^{n}\left(\sum_{x_{i j} \in \mathcal{X}_{\text {train }}}-y_{i j}^{k} \log \hat{y}_{i j}^{k}-\left(1-y_{i j}^{k}\right) \log \left(1-\hat{y}_{i j}^{k}\right)\right)$.

Different from the algorithm that considers PPI independently, GNN-PPI learns to combine protein neighbors to generate feature representations. Therefore, for the $\mathcal{X}_{\text {test }}$ constructed by our proposed BFS or DFS, GNN-PPI can also be based on its neighbors to generate suitable feature representations for multi-type PPI prediction. On the other hand, even if the PPI network $\mathcal{G}^{\prime}=\left(\mathcal{P}_{v}, \mathcal{X}_{\text {train }}\right)$ used in the training process is constructed with only $\mathcal{X}_{\text {train }}$, it can perform well for unknown PPI $x_{i j} \in \mathcal{X}_{\text {test. }}$ (See details in Table 4)

\section{Experiment}

\subsection{Dataset}

We use multi-type PPI data from the STRING database ${ }^{2}$ [Szklarczyk et al., 2019] to evaluate our proposed GNN-PPI. The STRING database collected, scored, and integrated most publicly available sources of protein-protein interaction information and built a comprehensive and objective global PPI network, including direct (physical) and indirect (functional) interactions. In this paper, we focus on the multi-type classification of PPI by STRING. It divides PPI into 7 types, namely reaction, binding, post-translational modifications (ptmod),

\footnotetext{
${ }^{2}$ https://string-db.org/
} 


\begin{tabular}{|c|c|c|c|c|c|c|c|c|}
\hline \multirow{2}{*}{ Dataset } & \multirow{2}{*}{$\begin{array}{l}\text { Partition } \\
\text { Scheme }\end{array}$} & \multicolumn{7}{|c|}{ Methods } \\
\hline & & SVM & RF & LR & DPPI & DNN-PPI & PIPR & GNN-PPI \\
\hline \multirow{3}{*}{ SHS27k } & Random & $75.35 \pm 1.05$ & $78.45 \pm 0.88$ & $71.55 \pm 0.93$ & $73.99 \pm 5.04$ & $77.89 \pm 4.97$ & $83.31 \pm 0.75$ & $\mathbf{8 7 . 9 1} \pm 0.39$ \\
\hline & BFS & $42.98 \pm 6.15$ & $37.67 \pm 1.57$ & $43.06 \pm 5.05$ & $41.43 \pm 0.56$ & $48.90 \pm 7.24$ & $44.48 \pm 4.44$ & $\mathbf{6 3 . 8 1} \pm 1.79$ \\
\hline & DFS & $53.07 \pm 5.16$ & $35.55 \pm 2.22$ & $48.51 \pm 1.87$ & $46.12 \pm 3.02$ & $54.34 \pm 1.30$ & $57.80 \pm 3.24$ & $\mathbf{7 4 . 7 2} \pm 5.26$ \\
\hline \multirow{3}{*}{ SHS148k } & Random & $80.55 \pm 0.23$ & $82.10 \pm 0.20$ & $67.00 \pm 0.07$ & $77.48 \pm 1.39$ & $88.49 \pm 0.48$ & $90.05 \pm 2.59$ & $92.26 \pm 0.10$ \\
\hline & BFS & $49.14 \pm 5.30$ & $38.96 \pm 1.94$ & $47.45 \pm 1.42$ & $52.12 \pm 8.70$ & $57.40 \pm 9.10$ & $61.83 \pm 10.23$ & $\mathbf{7 1 . 3 7} \pm 5.33$ \\
\hline & DFS & $58.59 \pm 0.07$ & $43.26 \pm 3.43$ & $51.09 \pm 2.09$ & $52.03 \pm 1.18$ & $58.42 \pm 2.05$ & $63.98 \pm 0.76$ & $\mathbf{8 2 . 6 7} \pm 0.85$ \\
\hline \multirow{3}{*}{ STRING } & Random & - & $88.91 \pm 0.08$ & $67.74 \pm 0.16$ & $94.85 \pm 0.13$ & $83.08 \pm 0.11$ & $94.43 \pm 0.10$ & $\overline{95.43} \pm 0.10$ \\
\hline & BFS & - & $55.31 \pm 1.02$ & $50.54 \pm 2.00$ & $56.68 \pm 1.04$ & $53.05 \pm 0.82$ & $55.65 \pm 1.60$ & $\mathbf{7 8 . 3 7} \pm 5.40$ \\
\hline & DFS & - & $70.80 \pm 0.45$ & $61.28 \pm 0.53$ & $66.82 \pm 0.29$ & $64.94 \pm 0.93$ & $67.45 \pm 0.34$ & $\mathbf{9 1 . 0 7} \pm 0.58$ \\
\hline
\end{tabular}

Table 1: Performance of GNN-PPI against comparative methods over different datasets and data partition schemes. The reported results are mean \pm std micro-averaged F1 score over three repeated experiments. Results of SVM on STRING is omitted for unafforable running time.

activation, inhibition, catalysis, and expression. Each pair of interacting proteins contains at least one of them. [Chen et al., 2019] randomly select 1,690 and 5,189 proteins from the Homo sapiens subset of STRING that shares $<40 \%$ of sequence identity to generate two subsets, namely SHS27k and SHS148k, which contain 7,624 and 44,488 multi-label PPIs. At the same time, we use all PPIs of Homo sapiens as our third dataset, namely STRING, which contains 15,335 proteins and 593,397 PPIs. We will use these three PPI datasets of different sizes to evaluate GNN-PPI and other PPI methods in the following content.

\subsection{Experimental Details}

\section{Experimental Settings and Metrics}

We select $20 \%$ of PPIs for testing, using our proposed BFS, DFS, and original evaluation (Random). The BFS or DFS partition algorithm has completely different results for different root nodes. To simulate the realistic scene mentioned in Section 3.3, the root node's degree should not be too large. We set the root node degree threshold $t=5$. To eliminate the influence of the randomness of data partitioning on the performance of PPI methods, we repeat experimental results under 3 different random seeds. We use the protein features based on amino acid sequence, refer to [Chen et al., 2019] using embedding method to represent each amino acid ${ }^{1}$. We adopt Adam algorithm [Kingma and $\mathrm{Ba}, 2014$ ] to optimize all trainable parameters. The other hyper-parameters settings are shown in the extended version ${ }^{1}$.

We evaluate the multi-label PPI prediction performance using micro-F1. This is because micro-averaging will emphasize the common labels in the dataset, which gives each sample the same importance. Since the different PPI types in the datasets we used are very imbalanced, micro-F1 may be preferred.

\section{Baselines}

We compare GNN-PPI against a variety of baselines, which can be categorized as follows:

1. Machine Learning based: We choose three representative machine learning (ML) algorithms, SVM [Guo et al., 2008], RF [Wong et al., 2015], and LR [Silberberg et al., 2014]. The input feature of the algorithms uniformly selects common handcrafted protein features, AC [Guo et al., 2008] and CTD [Du et al., 2017], of which CTD use seven attributes for the division ${ }^{1}$.
2. Deep Learning based: We choose three representative deep learning (DL) algorithms in PPI prediction, PIPR [Chen et al., 2019], DNN-PPI [Li et al., 2018] and DPPI [Hashemifar et al., 2018]. We construct the same architecture and hyper-parameters settings as the original papers and modify the output of the original implementation from a binary class to multi-label. The protein input feature based on the amino acid sequence is consistent with GNN-PPI.

\subsection{Results and Analysis}

Table 1 compares the performance of different methods under different evaluations and different datasets. Firstly, consider the impact of different evaluations, we can see that any method in Table 1 perform well under Random partition. However, under BFS or DFS partition, except for GNN-PPI, the performance of other methods declines clearly. Moreover, the performance under the DFS is generally higher than that of the BFS, which means that the clustered distribution of unknown proteins in the PPI network is harder to learn than discrete distribution. Next, observe the performance on different datasets. Regardless of the evaluations, the performance of any method will improve as the data size increases. However, the problems mentioned above will not be trivially solved by increasing the amount of data. Finally, comparing different methods, we can see that DL-based methods are generally better than ML-based, and GNN-PPI can achieve state-ofthe-art performance. However, under the Random partition, the advantage of GNN-PPI over DL-based methods will be smaller as the dataset size increases. The most prominent advantage of GNN-PPI is that under the BFS or DFS partition, and for the inter-novel-protein interactions, it can still learn useful feature representations from protein neighbors so as to obtain good performance in PPI prediction. In summary, the experimental results show that GNN-PPI can effectively improve the prediction accuracy of inter-novel-protein interactions. However, how to further push the performance to be comparable as Random partition is still a problem worthy of further discussion, and it is also our future work.

We make a more in-depth analysis of performance between PIPR and GNN-PPI on $\mathcal{X}_{\text {test }}$, as shown in Table 2. Observing the proportions of different subsets of the testset, we can find that under Random partition, more than $92 \%$ test samples belong to $\mathcal{X}_{\mathrm{BS}}$, which is consistent with our corollary 1 . PIPR performs well on the randomly divided testset (81.58 in 


\begin{tabular}{|c|c|c|c|c|c|c|c|c|c|c|c|c|}
\hline \multirow{2}{*}{ Dataset } & \multirow{2}{*}{$\begin{array}{l}\text { Partition } \\
\text { Scheme }\end{array}$} & \multicolumn{2}{|c|}{$\mathcal{X}_{\mathrm{BS}}$} & \multicolumn{2}{|c|}{$\mathcal{X}_{\mathrm{ES}}$} & \multicolumn{2}{|c|}{$\mathcal{X}_{\mathrm{NS}}$} & \multicolumn{5}{|c|}{$\mathcal{X}_{\text {Avg }}$} \\
\hline & & PIPR & GNN-PPI & PIPR & GNN-PPI & PIPR & GNN-PPI & Propo & $n(B S$ & /NS) & PIPR & GNN-PPI \\
\hline \multirow{3}{*}{ SHS27k } & Random & 83.12 & 88.31 & 64.48 & 74.28 & 35.29 & 33.33 & 92.2 & 7.5 & 0.3 & 81.58 & 87.11 \\
\hline & BFS & - & - & 44.92 & 68.08 & 30.34 & 46.25 & 0.0 & 72.6 & 27.4 & 40.92 & 62.10 \\
\hline & DFS & - & - & 58.25 & 72.22 & 48.77 & 63.22 & 0.0 & 88.6 & 11.4 & 57.17 & 71.19 \\
\hline \multirow{3}{*}{ SHS148k } & Random & 92.82 & 92.24 & 78.80 & 73.09 & 40.72 & 36.36 & 97.2 & 2.7 & 0.1 & 92.42 & 91.68 \\
\hline & BFS & - & - & 62.80 & 72.51 & 73.82 & 77.02 & 0.0 & 69.7 & 30.3 & 66.13 & 73.88 \\
\hline & DFS & - & - & 64.17 & 83.37 & 55.51 & 73.08 & 0.0 & 91.9 & 8.1 & 63.47 & 82.54 \\
\hline \multirow{3}{*}{ STRING } & Random & 94.32 & 95.42 & 61.65 & 77.68 & 33.33 & 57.14 & 99.7 & 0.3 & 0 & 94.23 & 95.37 \\
\hline & BFS & - & - & 56.71 & 83.99 & 39.87 & 72.83 & 0.0 & 85.8 & 14.2 & 54.31 & 82.41 \\
\hline & DFS & - & - & 68.61 & 90.38 & 55.22 & 87.07 & 0.0 & 94.3 & 5.7 & 67.84 & 90.19 \\
\hline
\end{tabular}

Table 2: In-depth analysis between PIPR and GNN-PPI over BS, ES and NS subsets.

\begin{tabular}{c|ccccc}
\hline \multirow{2}{*}{ Methods } & \multirow{2}{*}{ Trainset } & \multirow{2}{*}{ Testset } & \multicolumn{3}{c}{ Partition Scheme } \\
\cline { 4 - 6 } & & & Random & BFS & DFS \\
\hline \multirow{3}{*}{ PIPR } & \multirow{2}{*}{ SHS27k-Train } & SHS27k-Test & 81.58 & 40.92 & 57.17 \\
& & STRING & 42.79 & 48.55 & 57.44 \\
\cline { 2 - 6 } & \multirow{2}{*}{ SHS148k-Train } & SHS148k-Test & 92.42 & 66.13 & 63.47 \\
& & STRING & 53.85 & 63.74 & 62.46 \\
\hline \multirow{3}{*}{ GNN-PPI } & \multirow{2}{*}{ SHS27k-Train } & SHS27k-Test & 87.11 & 62.10 & 71.19 \\
\cline { 3 - 6 } & \multirow{2}{*}{ SHS148k-Train } & STRING & 66.85 & 66.39 & 67.43 \\
\cline { 3 - 6 } & & SHS148k-Test & 91.68 & 73.88 & 82.54 \\
& STRING & 73.12 & 67.43 & 70.64 \\
\hline
\end{tabular}

Table 3: Performance comparison of tested on trainset-homologous testset vs. unseen testset, under different evaluations.

SHS27k, 92.42 in SHS148k, and 94.23 in STRING), but if we further investigate the testset, we will find that PIPR performs very poorly for inter-novel-protein interactions $\left(x_{i j} \in \mathcal{X}_{\mathrm{ES}}\right.$ or $\mathcal{X}_{\mathrm{NS}}$ ), but it is dominated by $\mathcal{X}_{\mathrm{BS}}$, which has accurate performance and a high proportion. According to the results of Table 1 and Table 2, with sufficient $\mathcal{X}_{\mathrm{ES}}$ and $\mathcal{X}_{\mathrm{NS}}$ data, we can assert that the methods which treats PPI as an independent sample (represented by PIPR), cannot accurately predict inter-novel-protein interactions. On the contrary, our proposed GNN-PPI can still perform well under BFS and DFS. Moreover, as the data size increases, the performance of GNN-PPI is better (e.g., 82.41 vs. 54.31 in STRING-BFS and 90.19 vs. 67.84 in STRING-DFS).

Next, we study the ability of different evaluations to assess the model's generalization. We take the trained model's test performance on the larger dataset STRING as the model's true generalization ability. If the gap between the trainset-homologous test performance and the generalization is smaller, then the evaluation can better reflect the model's generalization. The experimental results are shown in Table 3. It can be seen that the previous evaluation (Random), whether it is for PIPR or GNN-PPI, the test performance on the STRING dataset has severely dropped. Like our speculation, it cannot reflect the generalization of the model. On the contrary, under the evaluation of BFS or DFS, its test performance can truly reflect the performance of the model, no matter it is good or bad (e.g. 66.13 vs. 63.74 in PIPR-SHS148kBFS and 71.19 vs. 67.43 in GNN-PPI-SHS27k-DFS). In fact, the testset obtained by BFS or DFS is theoretically the same as the sample tested on STRING. The only difference is the proportion of different types of PPI (BS, ES and NS). Testing

\begin{tabular}{ccccc}
\hline \multirow{2}{*}{$\begin{array}{c}\text { Partition } \\
\text { Scheme }\end{array}$} & \multirow{2}{*}{ Graph } & \multicolumn{3}{c}{ Dataset } \\
\cline { 3 - 5 } & & SHS27k & SHS148k & STRING \\
\hline \multirow{2}{*}{ BFS } & GCA & $63.81 \pm 1.79$ & $71.37 \pm 5.33$ & $78.37 \pm 5.40$ \\
& GCT & $60.61 \pm 5.32$ & $69.56 \pm 6.89$ & $73.23 \pm 3.93$ \\
\hline \multirow{2}{*}{ DFS } & GCA & $74.72 \pm 5.26$ & $82.67 \pm 0.85$ & $91.07 \pm 0.58$ \\
& GCT & $73.42 \pm 5.50$ & $80.35 \pm 2.20$ & $89.04 \pm 1.06$ \\
\hline
\end{tabular}

Table 4: Performance of GNN-PPI with different PPI Graph construction method.

On the STRING, the proportion of NS is higher.

Finally, we study the impact of the PPI network graph construction method (mentioned in 3.5) in the GNN-PPI. There are two graph construction methods, graph construct by all data $(\mathrm{GCA}, \mathcal{G}=(\mathcal{P}, \mathcal{X}))$ and graph construct by trainset $\left(\mathrm{GCT}, \mathcal{G}=\left(\mathcal{P}_{v}, \mathcal{X}_{\text {train }}\right)\right)$. The experimental results are shown in Table 4. It can be seen that the performance of GCA all exceeds that of GCT, which is reasonable because the graph construction of GCA accesses more complete information than GCT. Compared with BFS, in the case of DFS, the performance of GCT is closer to GCA, which seems to indicate that the protein neighbors are more complete, the performance will be better. What is more noteworthy is that GCT is still much higher than non-graph algorithms, which shows the superiority of GNN in processing the few-shot learning for multi-label PPI prediction task. Moreover, for unknown proteins, we often cannot know their neighbors in advance. The effectiveness of GCT shows that the trained model is robust to newly discovered proteins and their interactions.

\section{Conclusion}

In this paper, we study the significant performance degradation of existing PPI methods when tested in unseen dataset. Experimental results show that this problem is due to the poor performance of the model for inter-novel-protein interactions. However, current evaluation overlook the inter-novel-protein interactions, and are thus not instructive for the performance when tested on unseen datasets. Therefore, we design a new evaluation framework with two per-protein randomized data partition startegies, namely BFS and DFS, and propose a GNN based method GNN-PPI to model the correlations between PPIs. Our experimental results show that GNN-PPI outperforms state-of-the-art PPI prediction methods regardless of the evaluation is original or our proposed, especially for the inter-novel-protein interactions prediction. 


\section{References}

[Anfinsen, 1972] Christian B Anfinsen. The formation and stabilization of protein structure. Biochemical Journal, 128(4):737-749, 1972.

[Chen et al., 2019] Muhao Chen, Chelsea J-T Ju, Guangyu Zhou, Xuelu Chen, Tianran Zhang, Kai-Wei Chang, Carlo Zaniolo, and Wei Wang. Multifaceted protein-protein interaction prediction based on siamese residual rcnn. Bioinformatics, 35(14):i305-i314, 2019.

[De Las Rivas and Fontanillo, 2010] Javier De Las Rivas and Celia Fontanillo. Protein-protein interactions essentials: key concepts to building and analyzing interactome networks. PLoS Comput Biol, 6(6):e1000807, 2010.

[Deng et al., 2020] Yifan Deng, Xinran Xu, Yang Qiu, Jingbo Xia, Wen Zhang, and Shichao Liu. A multimodal deep learning framework for predicting drug-drug interaction events. Bioinformatics, 2020.

[Du et al., 2017] Xiuquan Du, Shiwei Sun, Changlin $\mathrm{Hu}$, Yu Yao, Yuanting Yan, and Yanping Zhang. Deepppi: boosting prediction of protein-protein interactions with deep neural networks. Journal of chemical information and modeling, 57(6):1499-1510, 2017.

[ERDdS and R\&wi, 1959] P ERDdS and A R\&wi. On random graphs i. Publ. math. debrecen, 6(290-297):18, 1959.

[Erdős and Rényi, 1960] Paul Erdôs and Alfréd Rényi. On the evolution of random graphs. Publ. Math. Inst. Hung. Acad. Sci, 5(1):17-60, 1960.

[Fields and Song, 1989] Stanley Fields and Ok-kyu Song. A novel genetic system to detect protein-protein interactions. Nature, 340(6230):245-246, 1989.

[Guo et al., 2008] Yanzhi Guo, Lezheng Yu, Zhining Wen, and Menglong Li. Using support vector machine combined with auto covariance to predict protein-protein interactions from protein sequences. Nucleic acids research, 36(9):3025-3030, 2008.

[Hashemifar et al., 2018] Somaye Hashemifar, Behnam Neyshabur, Aly A Khan, and Jinbo Xu. Predicting protein-protein interactions through sequence-based deep learning. Bioinformatics, 34(17):i802-i810, 2018.

[Kingma and Ba, 2014] Diederik P Kingma and Jimmy Ba. Adam: A method for stochastic optimization. arXiv preprint arXiv:1412.6980, 2014.

[Kipf and Welling, 2016] Thomas N Kipf and Max Welling. Semi-supervised classification with graph convolutional networks. arXiv preprint arXiv:1609.02907, 2016.

[Li et al., 2018] Hang Li, Xiu-Jun Gong, Hua Yu, and Chang Zhou. Deep neural network based predictions of protein interactions using primary sequences. Molecules, 23(8):1923, 2018.

[Nambiar et al., 2020] Ananthan Nambiar, Maeve Heflin, Simon Liu, Sergei Maslov, Mark Hopkins, and Anna Ritz. Transforming the language of life: Transformer neural networks for protein prediction tasks. In Proceedings of the 11th ACM International Conference on Bioinformatics, Computational Biology and Health Informatics, pages $1-8,2020$.

[Saha and others, 2020] Sriparna Saha et al. Amalgamation of protein sequence, structure and textual information for improving protein-protein interaction identification. In Proceedings of the 58th Annual Meeting of the Association for Computational Linguistics, pages 6396-6407, 2020.

[Shen et al., 2007] Juwen Shen, Jian Zhang, Xiaomin Luo, Weiliang Zhu, Kunqian Yu, Kaixian Chen, Yixue Li, and Hualiang Jiang. Predicting protein-protein interactions based only on sequences information. Proceedings of the National Academy of Sciences, 104(11):4337-4341, 2007.

[Silberberg et al., 2014] Yael Silberberg, Martin Kupiec, and Roded Sharan. A method for predicting protein-protein interaction types. PLoS One, 9(3):e90904, 2014.

[Skrabanek et al., 2008] Lucy Skrabanek, Harpreet K Saini, Gary D Bader, and Anton J Enright. Computational prediction of protein-protein interactions. Molecular biotechnology, 38(1):1-17, 2008.

[Sun et al., 2017] Tanlin Sun, Bo Zhou, Luhua Lai, and Jianfeng Pei. Sequence-based prediction of protein protein interaction using a deep-learning algorithm. BMC bioinformatics, 18(1):1-8, 2017.

[Szklarczyk et al., 2016] Damian Szklarczyk, John H Morris, Helen Cook, Michael Kuhn, Stefan Wyder, Milan Simonovic, Alberto Santos, Nadezhda T Doncheva, Alexander Roth, Peer Bork, et al. The string database in 2017: quality-controlled protein-protein association networks, made broadly accessible. Nucleic acids research, page gkw937, 2016.

[Szklarczyk et al., 2019] Damian Szklarczyk, Annika L Gable, David Lyon, Alexander Junge, Stefan Wyder, Jaime Huerta-Cepas, Milan Simonovic, Nadezhda T Doncheva, John H Morris, Peer Bork, et al. String v11: proteinprotein association networks with increased coverage, supporting functional discovery in genome-wide experimental datasets. Nucleic acids research, 47(D1):D607-D613, 2019.

[Wong et al., 2015] Leon Wong, Zhu-Hong You, Shuai Li, Yu-An Huang, and Gang Liu. Detection of protein-protein interactions from amino acid sequences using a rotation forest model with a novel pr-lpq descriptor. In International Conference on Intelligent Computing, pages 713720. Springer, 2015.

[Xu et al., 2018] Keyulu Xu, Weihua Hu, Jure Leskovec, and Stefanie Jegelka. How powerful are graph neural networks? arXiv preprint arXiv:1810.00826, 2018.

[Yang et al., 2020] Fang Yang, Kunjie Fan, Dandan Song, and Huakang Lin. Graph-based prediction of proteinprotein interactions with attributed signed graph embedding. BMC bioinformatics, 21(1):1-16, 2020. 